\title{
Interactive Props and Choreography Planning with the Mixed Reality Stage
}

\author{
Wolfgang Broll ${ }^{1}$, Stefan Grünvogel ${ }^{2}$, Iris Herbst ${ }^{1}$, Irma Lindt ${ }^{1}$, Martin Maercker ${ }^{3}$, \\ Jan Ohlenburg $^{1}$, and Michael Wittkämper ${ }^{1}$ \\ ${ }^{1}$ Fraunhofer-Institut für Angewandte Informationstechnik FIT, \\ Schloß Birlinghoven, 53754 Sankt Augustin, Germany \\ \{wolfgang.broll, iris.herbst, irma.lindt, jan.ohlenburg, \\ michael.wittkaemper\} afit. fraunhofer.de \\ 2 Laboratory for Mixed Realities, Institute at the Academy of Media Arts Cologne, \\ Schaafenstr. 25, D-50676 Köln, Germany \\ gruenvogel@lmr. khm. de \\ ${ }^{3}$ plan_b media ag, Schaafenstr. 25, 50676 Köln, Germany \\ maercker@planb_media.de
}

\begin{abstract}
This paper introduces-the Mixed Reality Stage-an interactive Mixed Reality environment for collaborative planning of stage shows and events. The Mixed Reality Stage combines the presence of reality with the flexibility of virtuality to form an intuitive and efficient planning tool. The planning environment is based on a physical miniature stage enriched with computergenerated props and characters. Users may load virtual models from a Virtual Menu, arrange those using Tangible Units or employ more sophisticated functionality in the form of special Tools. A major feature of the Mixed Reality stage is the planning of choreographies for virtual characters. Animation paths may be recorded and walking styles may be defined in a straightforward way. The planning results are recorded and may be played back at any time. User tests have been conducted that demonstrate the viability of the Mixed Reality Stage.
\end{abstract}

\section{Introduction}

During the planning process of an event such as a theatre performance, a concert or a product presentation the creativity and imagination of the people involved are in demand. Various ideas are discussed and revised or discarded. To illustrate an idea planners typically use sketches and/or specifically built 3D models. A common practice is to employ a downscaled model stage including props, real stage lights and jointed dolls representing actors. The physical models - usually at a ratio of 4:1 - are elementary components of the planning process and their arrangement visualizes the current planning status. Within the model stage planners can easily compose stage setups and test different lighting arrangements. However, physical models are not very flexible when it comes to the planning of more dynamic aspects of a stage event.

A higher flexibility in modeling the dynamic as well as the static aspects of a stage show may be reached by Virtual Reality technology. Typically, in VR planning tools, 
planners can choose from a large variety of virtual models that can be easily modified to fit into the stage setup. Animation tools are part of most VR systems and allow the modeling of changes in stage settings, choreographies or light shows. A stage show that was created with VR technology may give the spectator a good impression of the planning result. A disadvantage, however, is that VR technology is not very well suited for the planning process itself.

In order to support collaborative planning, a system needs to provide an appropriate user interface and it must respond to user interaction in real time. Many VR user interfaces do not facilitate the cooperative editing of virtual objects, nor do they consider a face-to-face collaboration among participants. In addition, the support of multiple users is often disappointing in terms of immersion (e.g. in CAVE-like environments). The real time capabilities of most VR systems are also very limited. A sufficient visualization of material properties is not yet feasible in real time. The same is true for professional lighting simulations which are often based on several hundred independent light sources. An example for a VR based planning environment that has successfully been used to plan stage shows is X-Rooms 0 . The systems uses stereoprojection and polarized filters to visualize $3 \mathrm{D}$ computer graphics and touch screen, joystick, mouse and steering wheel for interaction. A drawback of X-Rooms is the separation between working and presentation space, though.
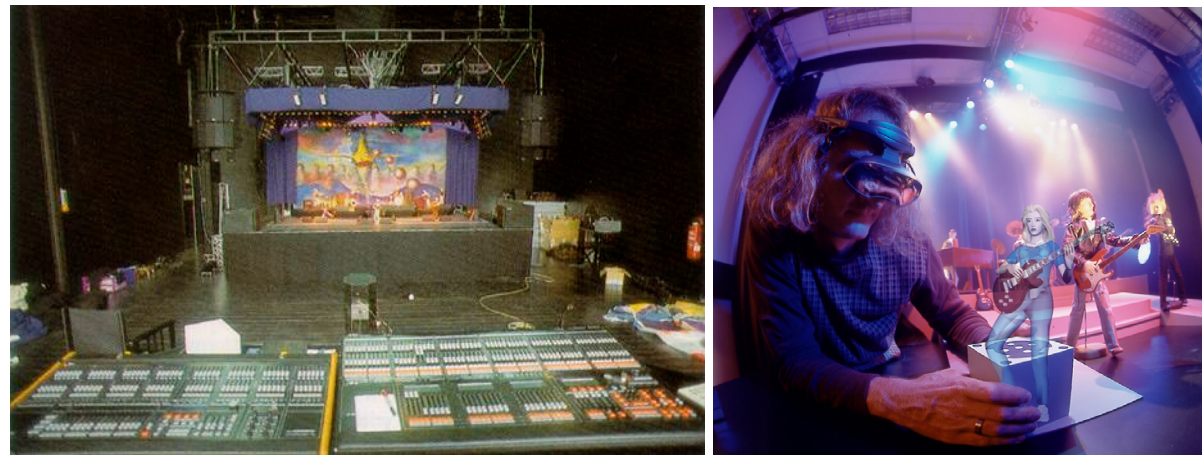

Fig. 1. The real model stage without virtual objects (left) and virtually enhanced as Mixed Reality Stage (right).

Mixed Reality planning tools, on the other hand, seamlessly combine real and virtual objects to overcome the individual limitations of real and virtual planning environments. Mixed Reality is a more viable technology for applications that require complex manipulation of three-dimensional information 0 . The Luminous Table project 0 , for example, supports urban planning using paper sketches as well as physical and virtual 3D models. Other Mixed Reality planning tools such as the AR Planning Tool 0 or the Build-It project 0 mainly use virtual objects as elementary planning components. Real objects serve as placeholders that are connected to virtual 3D models to form Tangible User Interfaces (TUIs) 0.

In the Mixed Reality Stage, physical models are used for a realistic presentation of typical components such as the stage itself, the props, or the stage lighting fixtures. The stage is extended by interactive computer graphics using Augmented Reality 
technology 0. Arbitrary virtual objects such as props or virtual characters are visualized and may easily be manipulated within the Mixed Reality Stage.

For each user the head position and its orientation are tracked 0 and the synthetic scene is visualized by means of semi-transparent Head Mounted Displays (HMDs). The point of view is egocentric, which serves to deepen the user's sense of immersion. A hybrid collaborative user interface was developed by employing a variety of techniques - such as TUI - where they best suited the different interaction tasks. A real control desk for stage machinery and a real stage lighting control board are also part of the user interface and allow planners to work in a familiar way without the need to learn new interaction mechanisms. With its intuitive user interface the Mixed Reality Stage provides multiple users with the means to creatively plan stage shows and to flexibly experiment with different ideas.

\section{The Mixed Reality Stage User Interface}

The Mixed Reality Stage is a collaborative AR environment 0 supporting face-to-face collaboration. Based on the positive experience and successful implementation of social protocols for group collaboration our interface approach focuses on providing powerful awareness mechanisms rather than limiting the user's freedom by rigid synchronization and locking mechanisms.

In cooperative planning situations a production design is analyzed from a high level perspective that follows from an institution's aesthetic and commercial strategies. Intricate details of a prop are, as a rule, not at issue. Therefore, users are primarily concerned with basic but expressive tasks such as arranging objects in space and time. The interaction design of the Mixed Reality Stage facilitates this approach by emphasizing simple, direct and reliable mechanisms to implement fundamental modeling operations:

- View Pointer: An object is selected when it is directly in a user's line of sight, i.e. users select objects by looking at them. A crosshair shown in the HMD helps users "aiming". The selection is visualized using the object's bounding box.

- Virtual Menu: Virtual objects have menus that contain those operations which are applicable to the specific type of object. The user opens a menu by issuing a single, modal voice command. Navigation in the menu hierarchy and invocation of operations is accomplished by selecting menu entries via the View Pointer and activating them by voice command.

- Tangible Units: A Tangible Unit (TU) realizes an interaction mechanism that provides direct and seamless manipulation of the position and orientation of a virtual object. A TU is composed of a tracked physical object (realoid) and an associated virtual object (virtualoid). To create a TU, the user collides the realoid with the virtualoid and links them by applying an explicit voice command. Another way to build a TU is to select the virtualoid, apply the command, select the realoid and apply a second command. The final voice command moves the virtualoid to the realoid and binds the two objects as a TU. This second method is provided as an alternative that obviates having to place the realoid at the same position as the virtualoid in order to form a TU. 
- Tools: The operations in a menu are tied to the virtualoid operand for which the menu was opened. Tools, on the other hand, are independent representations of individual operations in the workspace. They allow the user to configure an operation's parameters once, applying that operation to any number of virtualoids afterwards, and customizing the workspace by loading and positioning tools as desired. Tools can be made part of a Tangible Unit where the movement data is interpreted as parameters to the operation. Realized tools so far are: a scale tool to adjust the size of virtualoids, a texture tool to change the texture of a virtualoid, and time tools to control the playback of animations (e.g. play, stop, rewind, fast forward).

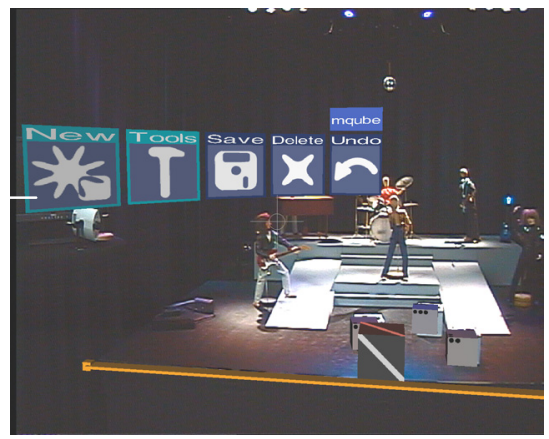

Fig. 2. Selecting an entry from a Virtual Menu using the View Pointer.

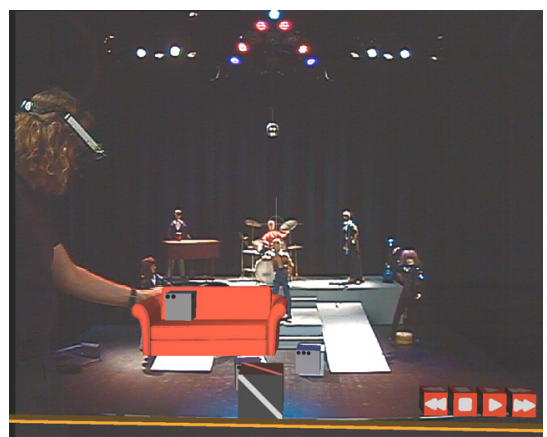

Fig. 3. Positioning a virtual couch that is part of a Tangible Unit. The Tools on the right side can be used to play back animations.

The use of voice commands is consciously limited to avoid the cognitive burden of having to learn many different symbols as well as to minimize interference with the communication between users. Voice commands are used solely to trigger functions and can be replaced by alternative trigger mechanisms, e.g. a pressed button of a wearable input device, depending on the individual requirements of the user or the particular environment. 


\section{Interaction with Virtual Characters}

A vital feature of the Mixed Reality Stage is the support for the development of choreographies. As a first step, planners create a rudimentary motion sequence for the actors. This can be used, for example, to verify whether an actor will reach a particular position within a certain time span or to prevent it from colliding with a prop while walking about the stage. As potential users made clear from preliminary requirements analysis onwards, real time animation of the character's motion is the decisive requirement for sophisticated choreography planning, playing a much greater role than the ability to render particular details of the animation (such as finger or face animation).

\subsection{Creating a Choreography}

The interaction mechanisms introduced in the previous section provide the basis for creating high level choreographies. Major tasks include the creation and assignment of walking paths and stage directions to a virtual character. To determine the path an actor should follow on the stage the planner links a virtual character to a TU. After activating the path recording operation from the character's menu, the movement of the TU is recorded. The same mechanism is used to finalize the path. The recorded path is displayed as a spline on the stage. The control points of the spline are displayed as well. The spline can be edited afterwards by linking individual control points to a TU.

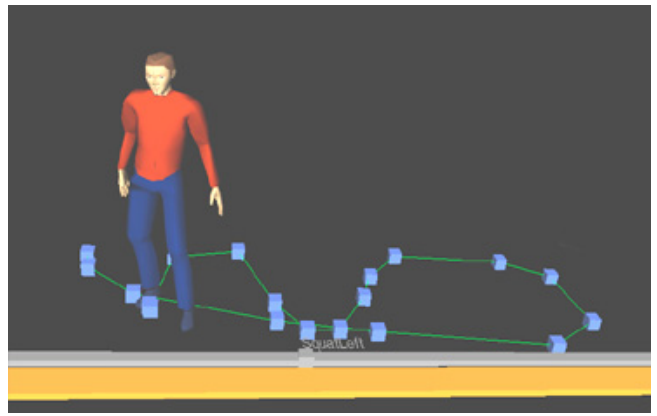

Fig. 4. Virtual character and its path (green line), control points (blue cubes) and assigned stage directions (grey bar and cube) in relation to time (orange bar and cube, cube not visible here)

Moreover, planners have to consider stage directions. Therefore, some basic movements for characters are provided. Planners are able to assign different walk styles, e.g. running. It is also possible to assign different basic gestures such as waving, pointing or squatting. These choreography elements are created using the character's virtual menu.

In order to correlate stage directions with time a time bar is displayed on which the current time is denoted by a virtual Now Point. In addition, a bar is displayed for each character with knobs that represent the stage directions given for that character. The 
position of the knob on the bar indicates when the character is to execute the stage direction. The knob can be part of a TU as well, enabling planners to move the characters' tasks in time. Fig. 4 shows a virtual character and additional handles for editing an existing choreography.

\subsection{Character Animations}

The creation and the editing of choreographies are implemented by a two-layered model. The upper layer is adjoin to the user interface component of the Mixed Reality Stage system. The upper layer is responsible for creating and deleting the characters and to manage their choreographies. The lower layer realizes the creation of the animations in real time 0. Each visible character is represented in the upper layer as an abstract object with reactive behavior, independent from the appearance or the skeleton of the character. If a stage direction is given via the user interface, a command is sent to the character which is responsible for the corresponding realization of the task in form of so called subtasks 0 .

During the editing of choreographies each character also resolves conflicts which can emerge between different tasks. For example, if a character that is walking receives the command to stand, a conflict results because both tasks (walk and stand) can not be executed at the same time. In principle it would be possible to warn the user, to point him to the conflict and to offer him alternatives to resolve the conflict. In the large majority of cases, however, the system can deduce the required modifications of a character's task by means of (configurable) heuristics and avoid a disruption of the users' workflow. Thus a walk cycle, for instance, is automatically stopped by the system if the user activates a task which results in a movement of the character in space (e.g. jumping).

Synchronization points are used to synchronize the character animation and the stage animation. They are user defined space-time constraints, i.e. they ensure that the character is at a specific position at a specific time. A character's task list settings may be such that they do not allow the character to immediately comply with newly set synchronization point constraints. The system then amends the task list of the character with special animation sequences. For example, if the character is too fast and reaches a synchronization point before the associated time then it waits and enters an idle animation loop. If it is too slow it does a comic-book-like "zip" to reach the required position at the designated time. Users can, of course, choose to modify the task list to reduce the role played by special animations in enabling the character to meet synchronization point constraints.

The subtasks realizing the tasks which are activated by the user interface (such as walking along a path) control the creation of the animation. For each character this is implemented at the lower layer by an animation engine. The animation engine gives access to dynamic motion models at the upper layer and sends the animation data to the skeleton of the character. Dynamic motion models are abstract descriptions of motions (e.g. walking) having their own sets of parameters. Examples for parameters are the style and the speed of walking or the direction for pointing. The parameters can be changed dynamically while the animations are created in real-time and the animations are then adapted dynamically. The animations are created by manipulation and blending of small pieces of pre-produced animation data 0 . 


\section{User Tests}

User tests were performed in the Mixed Reality Laboratory of Fraunhofer FIT. A Mixed Reality Stage based on a downscaled four-to-one model stage $(2 \mathrm{~m} \times 2 \mathrm{~m})$ was used. The tests were focused on the evaluation of the user interface mechanisms.

Seven people participated in the tests. All participants were from the theatrical preproduction domain with an average professional experience of 13 years. Two of them were permanent employees of (different) theatres, the other five were freelancers. They described their computer skills as above average, especially in regard to 3D modeling software.

In the beginning of each trial session the interaction mechanisms of the Mixed Reality Stage were introduced. The participants were then asked to describe typical problems from their everyday practice. These scenarios served as a foundation for experimentation within the individual areas of functionality as well as with the Mixed Reality Stage as a whole. The participants were assisted by a member of the project team as collaboration partner. The sessions closed with a discussion of the strengths and weaknesses of the system. During the tests interviewers took notes and the tests were videotaped. The sessions lasted two and a half hours on average. The main feedback received from these user tests was:

- Selecting objects with the view pointer was easy to understand and to use.

- The voice input to activate a selected command was facile to understand. The analogue to a mouse was obvious, and every participant controlled this interaction mechanism at once.

- The possibility to arrange virtual objects with a TU was novel for all participants and they were impressed when the virtual object followed the movements of the realoid. The participants readily accepted this mechanism for spatial operations and emphasized the sensual aspects of the interaction as playing an important role in their assessment.

- The participants appraised the creation of a character animation as a vital feature of the Mixed Reality Stage. The editing of animation splines using TUs was observed as straightforward and rated as being much easier than using a 3D modeling software.

\section{Conclusions and Future Work}

In this paper we presented a new approach for interactive props and choreography planning using the Augmented Reality environment Mixed Reality Stage. We introduced the individual interaction mechanisms with an emphasis on props and choreography planning support. Finally, we presented the initial results of ongoing user tests, confirming the validity of our approach. In our future work we plan to continue the evaluation with professional users and extend the user tests into field trials at different locations. The Mixed Reality user interface will be enhanced and adapted according to the feedback received. Additionally, we will investigate possibilities for using the technology and mechanisms in new application areas. 
Acknowledgements. This work is part of the mqube project and has been partially funded by the German Federal Ministry of Education and Research. We would like to thank the partners of the mqube project for their fruitful collaboration.

\section{References}

1. Azuma, R. T.: A Survey of Augmented Reality, Presence: Teleoperators and Virtual Environments 6, 4 (August 1997), 355-385

2. Billinghurst, M., Kato, H.: Collaborative Augmented Reality, Communication of the ACM Vol.45, No.7, 64-70 (2002)

3. Fitzmaurice, G., Ishii, H. Buxton, W. Bricks: Laying the Foundations for Graspable User Interfaces. Proceedings of the Conference on Human Factors in Computer Systems (CHI'95). 442-449 (1995)

4. Gausemeier, J., Fruend, J., Matysczok, C.: AR-Planning Tool - Designing Flexible Manufacturing Systems with Augmented Reality. Proceedings of Eurographics Workshop on Virtual Environments 2002

5. Grünvogel, S. Schwichtenberg, S.: Scripting Choreographies, T. Rist et. al. (Eds.), IVA 2003, Lecture Notes in Artificial Intelligence 2792, pp 170-174, 2003, Springer-Verlag Berlin Heidelberg, 2003

6. Grünvogel, S.: Dynamic character animations, International Journal of Intelligent Games \& Simulation Vol.2 No.1 (2003), pp. $11-19$

7. Isakovic, K., Dudziak, T., Köchy, K.: X-Rooms. A PC-based immersive visualization environment, Web 3D Conference, Tempe, Arizona, 2002.

8. Ishii, H., Ullmer, B.: Tangible Bits: Towards Seamless Interfaces between people, Bits and Atoms. CHI'97, Atlanta, Georgia 1997

9. Ishii, H., Underkoffler, J., Chak, D., Piper, B.: Augmented Urban Planning Work-bench: Overlaying Drawings, Physical Models and Digital Simulation. In Proceedings of ISMAR'02 (2002)

10. Krüger, H., Klingbeil, L., Kraft, E., Hamburger, R.: BlueTrak - A wireless six degrees of freedom motion tracking system. In Proceedings of ISMAR'03, Tokio, Japan, Okt. 2003

11. Rauterberg, M., Fjeld, M., Krüger, H., Bichsel, M., Leonhardt, U., Meier, M.: Build-IT: A Planning Tool for Construction and Design. In Proceedings of CHI'98 (1998)

12. Schmalstieg, D., Fuhrmann, A., Hesina, G., Szalavari, Z., Encarnação, M., Gervautz. M., Purgathofer, W.: The Studierstube Augmented Reality Project. In PRESENCE Teleoperators and Virtual Environments, MIT Press, 2002 